\title{
Prominent Non-Motor Symptoms in Patients with Parkinson's Disease and Pain
}

Cheng-Jie Mao ${ }^{1,2}$, Ju-Ping Chen ${ }^{3}$, Xiao-Yi Zhang ${ }^{1}$, Jie Li ${ }^{1,2}$, Kang-Ping Xiong ${ }^{1,2}$, Yi Chen ${ }^{1}$, Si-Jiao Li ${ }^{1}$, Wei-Dong Hu ${ }^{1}$, Li-Fang Hu${ }^{2}$ and Chun-Feng Liu ${ }^{1,2^{*}}$

${ }^{1}$ Department of Neurology, the Second Affiliated Hospital of Soochow University, Suzhou, China

${ }^{2}$ Institute of Neuroscience, Soochow University, Suzhou, China

${ }^{3}$ Department of Neurology, Hospital of Changshu Traditional Chinese Medicine, East Huanghe Road, Changshu, China

*Corresponding author: Chun-Feng Liu, Department of Neurology, the Second Affiliated Hospital of Soochow University, 1055, Sanxiang Road, Suzhou, China, Tel: + 86512 67783307; Fax: +86 512 68284303; E-mail : liucf@suda.edu.cn

Received date: Apr 17, 2014, Accepted date: May 24, 2014, Published date: May 30, 2014

Copyright: (c) 2014 Mao JC, et al. This is an open-access article distributed under the terms of the Creative Commons Attribution License, which permits unrestricted use, distribution, and reproduction in any medium, provided the original author and source are credited.

\begin{abstract}
Sensory disorders including pain and other non-motor symptoms occur frequently in patients with Parkinson's disease (PD). Studies on the correlation between pain and non-motor symptoms such as depression and sleep disorders are relatively more, so further investigations of the correlation between pain and other non-motor symptoms of PD are needed. A total of 142 patients with PD with or without pain were included in the study. PD severity was evaluated using the Unified Parkinson's Disease Rating Scale (UPDRS) and the Hoehn and Yahr (H/Y) stage scale. Pain severity was analyzed using the Visual Analog Scale (VAS) and the Brief Pain Inventory (BPI). The Hamilton Rating Scale for Depression (HRSD; 24 items), Montreal Cognitive Assessment Beijing Version (MoCA), and non-motor questionnaire NMSQT) measured symptoms of depression, cognitive function, and nonmotor symptoms. The incidence of pain was $47.9 \%$ in patients with PD, most of whom had moderate pain levels. Patients with pain showed higher HRSD, UPDRS, H/Y, and NMSQT scores and lower MoCA scores compared to patients without pain. HRSD and NMSQT scores were closely related with pain $(p<0.001)$. The incidence of pain is higher in patients with $P D$ in pain than in those without pain. Musculoskeletal pain was commonly seen in patients with PD. Compared to controls and patients with PD without pain, non-motor symptoms were more prominent in patients with pain.
\end{abstract}

Keywords: Non-motor symptoms; Parkinson's disease; Pain; Depression; Manifestation; Predictors

\section{Introduction}

Non-motor symptoms of Parkinson's disease (PD) have received increasingly more recognition in recent years. Pain is among such non-motor sensory disorders and occurs frequently in patients with PD. Although chronic pain among patients with PD can be caused by motor symptoms, it has also been reported independently of these symptoms [1]. Extensive co morbidity has been reported between chronic pain syndromes and mood and anxiety disorders [2,3]. Dysfunction in endogenous pain inhibition caused by a dopaminergic deficiency in the basal ganglia, particularly in the striatum and mesolimbic areas, is the main pathophysiological mechanism involved in PD-associated nociceptive abnormalities [2,4]. In addition to the anatomic overlap between the brain regions associated with pain processing and those that comprise the dopamine system, there is substantial overlap between the cognitive and affective functions influenced by dompaminergic (DA) neurotransmission. DA neurotransmission plays an important role in outcome prediction, attention, response inhibition, and motivation [5] as well as in affective symptoms associated with anxiety [6,7] and depression [8].

Cognitive and affective factors also influence the subjective experience of pain. Depression may be one of the leading factors contributing to pain [9]. One study on the relationship between pain and olfactory dysfunction, the two most common sensation dysfunctions in patients with $\mathrm{PD}$, showed that pain processing in these patients was impaired and significantly correlated with smell dysfunction [10]. Research on the relationship between cognitive dysfunction and non-motor symptoms in patients with PD has shown that sleep disturbances in this group may be an early marker of dementia [11]. Therefore, the relationships between non-motor symptoms in patients with PD are complex and multiplex. Previous research on pain and other non-motor symptoms in patients with $\mathrm{PD}$ has mainly focused on the relationship between pain and depression. Other than depression, the relationships between pain and other nonmotor symptoms in these patients are not yet known.

The aims of the study were to: examine the pain frequency and clinical characteristics of pain and examine the relationship between pain and other non-motor symptoms; and identify and analyze the factors that are independently associated with pain in patients with PD.

\section{Patients and Methods}

\section{General clinical characteristics and questionnaires}

A total of 142 consecutive patients with PD (82 men and 60 women) who were recruited from the outpatient clinic of our hospital participated in the study. The clinical diagnosis of PD matched the United Kingdom Parkinson's Disease Society Brain Bank (UKPDSBB) criteria [12]. Patients who had undergone deep brain stimulation, who were showing clinical or electrophysiological evidence of peripheral neuropathy or a disease that might be associated with pain such as diabetes mellitus, or Parkinsonism-plus syndrome, epilepsy or other neurological disease, or severe dementia (Montreal Cognitive Assessment Beijing Version $[\mathrm{MoCA}]<10$ ) were excluded from the study. A questionnaire was designed to collect 
Page 2 of 6

patients' demographic data and other information including history of onset, medical treatment, and features of motor disability, motor fluctuations, and dyskinesia. Symptoms of depression, cognitive function, and non-motor symptoms in patients with $\mathrm{PD}$ were evaluated using the Unified Parkinson's Disease Rating Scale (UPDRS) [13], Hoehn and Yahr Stage (H/Y) [14], Hamilton Depression Rating Scale (HRSD; 24 items) [15], MoCA [16], and non-motor questionnaire (NMS Quest) [17]. The number of non-motor symptoms according to the NMS Quest was recorded as the NMSQT. At the time of the examination, the patients were asked to report any pain they had experienced for at least 3 months. Average pain was evaluated using the Visual Analog Scale (VAS; scale, 0-100) [18] and the Brief Pain Inventory (BPI; scale, 0-10). BPI contains seven interference items including general activity, mood, walking ability, normal work, and relationships with other people, sleep, and enjoyment of life [19]. A total of 54 healthy control subjects from our medical center also completed the NMS Quest, and the results were compared between groups.

All patients were at the "on" stage during the interview except those who were not being treated with medications. Daily equivalent levodopa dosage was calculated for those patients receiving treatments. To analyze the drug effect on the pain of patients with $\mathrm{PD}$, the levodopa equivalent dose was calculated according to the following conversion formula: regular levodopa dose $\times 1+$ slow release levodopa $\times 0.75+$ bromocriptine $\times 10+$ apomorphine $\times 10+$ ropinirole $\times 20+$ pergolide $\times 100+$ piribedil $\times 1+$ pramipexole $\times 100+$ selegiline $\times 10$ if taking entacapone $+[$ regular levodopa dose + (slow release levodopa $\times$ $0.75)] \times 0.2$ were calculated [20]. Patient characteristics are shown in Table 1.

\begin{tabular}{|c|c|c|c|c|}
\hline & $\begin{array}{l}P D \text { with pain } \\
n=68\end{array}$ & $\begin{array}{l}P D \quad \text { without } \\
\text { pain } \\
n=74\end{array}$ & $\begin{array}{l}\text { Control } \\
n=54\end{array}$ & $P$ value \\
\hline Age (years)a & $66.85 \pm 9.54$ & $69.61 \pm 10.17$ & $\begin{array}{l}68.39 \pm 7.8 \\
2\end{array}$ & 0.346 \\
\hline Gender (male \%)b & $39(57.4)$ & $43(58.1)$ & $30(55.6)$ & 0.155 \\
\hline $\begin{array}{l}\text { Disease } \\
\text { (months)a }\end{array}$ & $69.18 \pm 48.30$ & $71.65 \pm 34.10$ & & 0.723 \\
\hline Age onset (years)a & $61.07 \pm 9.61$ & $63.64 \pm 10.12$ & & 0.125 \\
\hline UPDRS I a & $3.66 \pm 2.33$ & $1.95 \pm 1.68$ & & 0.000 \\
\hline UPDRS II a & $12.57 \pm 6.10$ & $9.04 \pm 4.94$ & & 0.000 \\
\hline UPDRSIIIa & $25.13 \pm 12.60$ & $20.27 \pm 10.95$ & & 0.015 \\
\hline UPDRSIVa & $2.18 \pm 2.47$ & $0.92 \pm 1.50$ & & 0.000 \\
\hline $\mathrm{H} / \mathrm{Y}$ stagea & $2.33 \pm 0.76$ & $1.95 \pm 0.80$ & & 0.005 \\
\hline HRSDa & $15.54 \pm 8.82$ & $7.41 \pm 6.22$ & & 0.000 \\
\hline MoCAa & $22.49 \pm 4.77$ & $23.89 \pm 3.09$ & & 0.037 \\
\hline NMSQTa & $8.38 \pm 4.87$ & $4.61 \pm 3.16$ & & 0.000 \\
\hline $\begin{array}{lr}\text { Daily } & \text { equivalent } \\
\text { levodopa } & \text { dosage } \\
\text { (mg)a } & \end{array}$ & $313.92 \pm 189.50$ & $\begin{array}{l}375.35 \pm 250.1 \\
0\end{array}$ & & 0.104 \\
\hline $\begin{array}{l}\text { Motor } \\
\text { (yes)c }\end{array}$ & 33 & 17 & & 0.001 \\
\hline
\end{tabular}

\begin{tabular}{|l|l|l|l|l|}
\hline Dyskinesias (yes)c & 16 & 6 & & 0.011 \\
\hline
\end{tabular}

Table 1: Clinical features of patients and univariate statistical analysis, ${ }^{a}$ means and standard deviations; ${ }^{b}$ percentages; ${ }^{c}$ number of patients say "yes".

\section{Pain definition in patients with PD}

$\mathrm{PD}$-associated pain was divided into five categories according to Ford B [21]: musculoskeletal, dystonic, radicular-neuropathic, central, and akathitic pain. Other pain syndromes, such as peripheral neuropathic pain and oral and genital pain, may occur more frequently in the presence of $\mathrm{PD}$ and, as such, were recognized as other types of discomfort according to the review by Jankovic J $[22,23]$. The aching, cramping, and joint pain in patients with PD commonly result from a lack of mobility in the affected limbs and joints, deformities of posture, stiffness of limb movements, and awkward gait mechanics. Musculoskeletal pain tends to increase as the duration of PD increases. Frozen shoulder is one of the most common causes of musculoskeletal pain. Radicular or neuritic pain was well localized to the territory of a nerve or nerve root. Dystonic pain was associated with sustained twisting movements and postures and seemed to be closely related to medication dosing. Central pain was described as feelings of burning, tingling, formication, and "neuropathic" sensations that were often relentless and bizarre in quality and with or without an autonomic character with visceral sensations or dyspnea. Akathitic pain or other discomfort was considered inner restlessness, an urge to move, the presence of oral and genital pain, and burning mouth or vagina syndrome. This type of pain may represent a sensory wearing off and improve with levodopa.

\section{Statistical analysis}

Statistical analyses were performed using SPSS 10.0 statistical analysis software (SPSS Inc. Chicago, IL, USA). Chi-square and student t-tests were used to compare constituent ratios and means in patients with and without pain and in controls. A logistic regression model was conducted to examine the predictors of pain. Bivariate correlations about gender, age, disease duration, age onset, score of UPDRS I, UPDRS II, UPDRSIII, UPDRSIV , HRSD, MoCA, and NMSQT score, motor fluctuation, dyskinesia, and the equivalent dosage of levodopa were analyzed between patients with and without pain. Each UPDRS score, H/Y stage, HRSD, MoCA, and NMSQT score, motor fluctuation, and dyskinesia was included in the covariance model. Values of $\mathrm{P}<0.05$ were defined as statistically significant.

The regional ethics committee of Soochow University approved the study. Informed consent was obtained from each patient.

\section{Results}

\section{Subject characteristics}

A total of 142 patients with PD but without dementia and 54 healthy control subjects were included in the study (Table 1). Patients and controls were age and gender-matched. The control population's mean age was 68.4 years, and $55.6 \%(30 / 54)$ were men. The patients' mean age was 68.3 years, and $57.4 \%$ were men (Table 1 ). Mean age at onset was 62.4 years and the average PD duration was 70.5 months with a median $\mathrm{H} / \mathrm{Y}$ stage of 2.1. Mean scores for each part of the 
UPDRS were 2.8, 10.7, 22.6, and 1.5. Mean HRSD and MoCA scores were 11.3 and 23.22, respectively. According to the NMSQ, there was a mean of 6.4 occurrences of non-motor symptoms. In all of the patients with $\mathrm{PD}$, the mean daily equivalent levodopa dosage was $343.3 \mathrm{mg}$. Motor fluctuation was present in $35.2 \%$ of the sample and the prevalence of dyskinesia was $15.5 \%$.

A total of $47.9 \%(68 / 142)$ of the patients with PD had pain symptoms. The difference in disease duration between the two groups was not statistically significant. However, patients with pain had higher scores on each part of the UPDRS and a higher H/Y stage than did patients without pain. The mean HRSD score in patients with PD and pain was 15.5; this was higher than the mean score of 7.4 in patients with PD but without pain. In patients with $\mathrm{PD}$ and pain, the mean MoCA and NMSQT scores were 22.5 and 8.4, respectively. In patients with PD but without pain, the mean MoCA and NMSQT scores were 23.9 and 4.6, respectively. Differences in these items were significant between the two groups $(\mathrm{P}=0.037, \mathrm{P}=0.000)$. The prevalence of motor fluctuation and dyskinesia was $48.5 \%(33 / 68)$ and $23.5 \%(16 / 68)$ in patients with pain and $23.0 \%(17 / 74)$ and $8.1 \%(6 / 74)$ in patients without pain.

\section{Clinical types of pain in PD patients}

PD-associated pain was recorded in $47.9 \%$ (68/142) of cases. Of these, $47.1 \%(32 / 68)$ of patients with PD and pain had musculoskeletal pain. Dystonic pain occurred in 23.5\% (16/68) of patients with PD and pain, while neuropathic pain, central pain, akathitic discomfort, and other pain was present in $19.1 \%(13 / 68), 7.4 \%(5 / 68)$, and $2.9 \%(2 / 68)$, respectively. Nine patients (13.2\%) reported more than one type of pain. The mean VAS score was 5.79. The mean BPI scores for seven interference items were 4.53 (general activity), 4.65 (mood), 4.66 (walking ability), 5.00 (normal work), 2.71 (relationships with other people), 3.76 (sleep), and 4.56 (enjoyment of life).

\section{Non-motor manifestations in patients with PD with and without pain}

Completed NMS Quest data were compared between patients with and without pain and to the control population (Table 2). Not surprisingly, the tenth item (pain presence percentage) was significantly different since patients with $\mathrm{PD}$ were divided according to pain status but controls were not. Constipation $(55.9 \%, 38 / 68)$ was the most prominent non-motor symptom in patients with $\mathrm{PD}$ and pain, followed by memory impairment $(51.5 \%, 35 / 68)$. The least common symptom was delusions. Patients with $\mathrm{PD}$ and pain had higher incidences of weight loss, memory disturbances, depression, insomnia, restless leg syndrome, and swelling than did patients without pain ( $\mathrm{P}=0.003, \mathrm{P}=0.000, \mathrm{P}=0.048, \mathrm{P}=0.003, \mathrm{P}=0.043, \mathrm{P}=0.016$, respectively). Comparisons between $\mathrm{PD}$ patients with pain and control subjects showed that patients with $\mathrm{PD}$ and pain were more likely to experience dribbling, loss of taste or smell, swallowing and choking difficulties, constipation, incomplete bowel emptying, weight loss, memory loss, loss of interest, difficulty concentrating, sad or blue mood, anxiety, decreased sex drive, insomnia, intense and vivid dreams, acting out during dreams, and swelling $(\mathrm{P}=0.012, \mathrm{P}=0.001, \mathrm{P}=0.002, \mathrm{P}=0.000$, $\mathrm{P}=0.017, \mathrm{P}=0.014, \mathrm{P}=0.045, \mathrm{P}=0.000, \mathrm{P}=0.000, \mathrm{P}=0.000, \mathrm{P}=0.017$, $\mathrm{P}=0.015, \mathrm{P}=0.017, \mathrm{P}=0.018, \mathrm{P}=0.000, \mathrm{P}=0.001, \mathrm{P}=0.041$, respectively). Results comparing the patients without pain to control subjects were similar for all above symptoms with the exception of weight loss, memory loss, anxiety, insomnia, and swelling $(\mathrm{P}=0.822, \mathrm{P}=0.194$, $\mathrm{P}=0.096, \mathrm{P}=0.733, \mathrm{P}=0.887$, respectively). Constipation $(54.1 \%, 40 / 74)$ was also the most prominent non-motor symptom in patients with $\mathrm{PD}$ but without pain, followed by nocturia $(31.1 \%, 23 / 74)$, although its incidence was not significantly different from that of control subjects. The least common symptom was also delusions.

\begin{tabular}{|c|c|c|c|c|c|c|}
\hline & $\begin{array}{l}\text { PD-pain(+) } \\
(n=68) \text { yes (\%) }\end{array}$ & $\begin{array}{l}\text { PD- pain(-) } \\
(n=74) \text { yes (\%) }\end{array}$ & $\begin{array}{l}\text { Control } \\
\text { (n=54)yes (\%) }\end{array}$ & P 1 value & P2 value & P3 value \\
\hline 1. Dribbling & $13(19.1)$ & $15(20.3)$ & $2(3.7)$ & 0.863 & 0.012 & 0.006 \\
\hline 2. Loss of Taste/Smell & $18(26.5)$ & $14(18.9)$ & $2(3.7)$ & 0.282 & 0.001 & 0.010 \\
\hline 3. Swallowing/Choking difficulties & $5(7.4)$ & $11(14.9)$ & $1(1.9)$ & 0.268 & 0.002 & 0.013 \\
\hline 4. Nausea/Vomiting & $3(4.4)$ & $2(2.7)$ & $3(5.6)$ & 0.581 & 0.772 & 0.411 \\
\hline 5. Constipation & $38(55.9)$ & $40(54.1)$ & $9(16.7)$ & 0.827 & 0.000 & 0.000 \\
\hline 6. Bowel incontinence & $2(2.9)$ & $1(1.4)$ & $1(1.9)$ & 0.510 & 0.700 & 0.822 \\
\hline 7. Bowel emptying incomplete & 16(23.5) & $16(21.6)$ & $4(7.4)$ & 0.786 & 0.017 & 0.029 \\
\hline 8. Urgency & $17(25.0)$ & $14(18.9)$ & $10(18.5)$ & 0.381 & 0.392 & 0.954 \\
\hline 9. Nocturia & $20(29.4)$ & $23(31.1)$ & $9(16.7)$ & 0.829 & 0.100 & 0.063 \\
\hline 10. Pains & $32(47.1)$ & $0(0)$ & $16(29.6)$ & 0.000 & 0.050 & 0.000 \\
\hline 11. Weight loss & $10(14.7)$ & $1(1.4)$ & $1(1.9)$ & 0.003 & 0.014 & 0.822 \\
\hline 12. Remembering & $35(51.5)$ & $17(23.0)$ & $18(33.3)$ & 0.000 & 0.045 & 0.194 \\
\hline 13. Loss of interest & $23(33.8)$ & $15(20.3)$ & $0(0.0)$ & 0.068 & 0.000 & 0.000 \\
\hline 14. Halucinations Delusions & $6(8.82)$ & $4(5.4)$ & $1(1.9)$ & 0.426 & 0.100 & 0.305 \\
\hline
\end{tabular}




\begin{tabular}{|l|l|l|l|l|l|l|}
\hline 15.Concentrating & $15(22.1)$ & $8(10.8)$ & $0(0.0)$ & 0.069 & 0.000 & 0.013 \\
\hline 16. Sad, blues & $26(38.2)$ & $17(23.0)$ & $3(5.6)$ & 0.048 & 0.000 & 0.007 \\
\hline 17. Anxiety & $14(20.6)$ & $11(14.9)$ & $3(5.6)$ & 0.371 & 0.017 & 0.096 \\
\hline 18. Sex_drive & $5(7.4)$ & $3(4.1)$ & $2(3.7)$ & 0.394 & 0.015 & 0.013 \\
\hline 19. Sex_difficulty & $7(10.3)$ & $2(2.7)$ & $2(3.7)$ & 0.064 & 0.017 & 0.012 \\
\hline 20. Dizziness & $18(26.5)$ & $13(17.6)$ & $8(14.8)$ & 0.200 & 0.118 & 0.626 \\
\hline 21. Falling & $8(11.7)$ & $12(16.2)$ & $3(5.6)$ & 0.446 & 0.234 & 0.064 \\
\hline 22. Daytime sleepiness & $11(16.2)$ & $10(13.5)$ & $3(5.6)$ & 0.655 & 0.068 & 0.141 \\
\hline 23. Insomnia & $26(38.2)$ & $12(16.2)$ & $10(18.5)$ & 0.003 & 0.018 & 0.733 \\
\hline 24. Intense, vivid dreams & $29(42.7)$ & $21(28.4)$ & $5(9.3)$ & 0.075 & 0.000 & 0.008 \\
\hline 25. Acting out during dreams & $20(29.4)$ & $22(29.7)$ & $3(5.6)$ & 0.967 & 0.001 & 0.001 \\
\hline 26. Restless legs & $23(33.8)$ & $14(18.9)$ & $11(20.4)$ & 0.043 & 0.100 & 0.838 \\
\hline 27. Swelling & $14(20.6)$ & $5(6.8)$ & $4(7.4)$ & 0.016 & 0.041 & 0.887 \\
\hline 28. Sweating & $20(29.4)$ & $15(20.3)$ & $8(14.8)$ & 0.207 & 0.057 & 0.427 \\
\hline 29. Diplopia & $6(8.8)$ & $3(4.1)$ & $3(5.6)$ & 0.244 & 0.493 & 0.691 \\
\hline 30. Delusions & 0 & 0 & constant & constant & constant \\
\hline
\end{tabular}

Table 2: Percentage of non-motor manifestations in PD patients with and without pain and in controls, P1: differences between PD patients with pain and without pain; P2: differences between PD patients with pain and controls; P3: differences between PD patients without pain and controls.

\section{Independent predictors of pain in patients with PD}

A logistic regression model between pain and variables was established to confirm the independent predictors of pain in patients with PD. These variables included gender, age, disease duration, age at onset, location of onset, onset symptoms, UPDRS I, UPDRS II, UPDRS III, and UPDRS IV scores, H/Y stage, dyskinesia, motor fluctuations, HRSD score, MoCA score, daily equivalent levodopa dosage, and NMSQT. UPDRS I, UPDRS II, UPDRS III, and UPDRS IV, H/Y stage, dyskinesia, motor fluctuation, HRSD, MoCA, and NMSQT scores were included in the logistic regression model.

HRSD and NMSQT scores were entered into the model. On multivariable analysis, HRSD $(\mathrm{P}=0.000)$ and NMSQT $(\mathrm{P}=0.012)$ scores were associated with pain in patients with PD (Table 3 ). The HosmerLemeshow goodness-of-fit test supported the validity of our regression model.

\begin{tabular}{|l|l|l|l|l|l|}
\hline Variable & B & SE & Wald & P value & $\mathbf{9 5 \% C l}$ \\
\hline NMSQT & -0.196 & 0.078 & 6.338 & 0.012 & $\begin{array}{l}0.705 ~ 0.95 \\
7\end{array}$ \\
\hline HRSD & -0.133 & 0.038 & 12.138 & 0.000 & $\begin{array}{l}0.813 \sim 0.94 \\
4\end{array}$ \\
\hline
\end{tabular}

Table 3: Logistic regression model examining predictors of pain, Abbreviations: $\mathrm{B}=\mathrm{B}$ coefficient; $\mathrm{SE}=$ standard error.

\section{Discussion}

Although there has been a lot of research on pain and other nonmotor symptoms, particularly depression, in patients with PD [24,25], the unique aspect of the current study was the correlation between pain and an extensive range of other non-motor symptoms. This was accomplished through the use of NMS Quest and by comparing responses on the NMS Quest questionnaire between patients with PD with and without pain and controls. The aim of the study was to observe the pain characteristics and examine the relationship between pain and other non-motor symptoms as well as factors that are independently associated with pain in patients with $\mathrm{PD}$.

The prevalence of all types of $\mathrm{PD}$-associated pain has been shown to be $24-83 \%$ [22,23]. However, we cannot conclude whether the pain is the result of dysfunction in basal ganglia pathway or the deterioration of clinical manifestations. In our study, the prevalence of pain in patients with PD was $47.9 \%$ (68/142). Musculoskeletal pain was the most common type of pain occurring in Chinese patients with PD, similar to the results of other studies $[4,24,26]$. The prevalence of musculoskeletal pain in the current study was $47.1 \%(32 / 68)$, while the prevalence of dytonic, neuropathic, central, and akathitic discomfort and other pain was $27.9 \%(19 / 68), 22.1 \%(15 / 68), 10.3 \%(7 / 68)$, and $5.9 \%(4 / 68)$, respectively. Nine patients (13.2\%) described more than one type of pain. VAS and BPI were completed simultaneously. Pain in patients with PD is generally relatively mild or moderate [27]. The mean VAS (1-100) score was 57.9 in our study.

The patients' clinical characteristics showed that those with PD and pain had higher scores on each part of the UPDRS and a higher H\&Y 
stage than did those without pain. Patients with pain had more severe PD symptoms. Patients with pain may progress more rapidly than patients without pain because disease duration did not differ between the two groups, but clinical symptoms were more severe in patients with pain than in patients without pain. Lack of mobility may also contribute to the occurrence of pain in patients with PD. Mean HRSD, MoCA, and NMSQT scores were 15.5, 22.5, and 8.4 in patients with pain and 7.41, 23.9, and 4.6 in those without. These findings in patients with PD suggested that patients with pain were more likely to be depressed and have a cognitive impairment and a corresponding increase in non-motor symptoms related to pain occurrence [28].

The daily equivalent levodopa dosage was $313.9 \mathrm{mg}$ in patients with PD and pain. This was not higher than that in patients without pain supposing that the use of anti-PD medications may not play a role in pain occurrence. Motor fluctuation was present in $48.5 \%$ of the PD patients with pain and the presence of dyskinesia was $23.5 \%$ in these patients. The frequency of motor fluctuation and dyskinesia was obviously higher in patients with pain than in patients without pain, indicating that dysfunction of the dopamine circuit may play a role in pain occurrence in patients with PD. Sensitization phenomena may explain the role of dyskinesia and motor fluctuations in patients with PD and pain. Lim SY et al. [29] considered that plasticity changes may also occur in pain responses in patients with dyskinesia.

To examine whether pain is related to other non-motor symptoms in patients with PD, the NMS Quest scale was used in this study. The NMS Quest is a useful clinical tool for screening non-motor symptoms in patients with PD [30]. These patients had a higher frequency of non-motor symptoms than did the controls. Constipation was the most common non-motor symptom in patients with PD regardless of pain status. The second most common non-motor symptom in patients with pain was memory impairment, while in patients without pain, the second most common non-motor symptom was nocturia. Delusion was not seen in any of the patients. These results suggest that patients with PD have a higher frequency of non-motor symptoms than control subjects, especially gastrointestinal, sensory, sleep disorders, autonomic, neuropsychiatric, cognitive impairment, weight loss, restless legs, and swelling. More severe degrees of weight loss, memory loss, depression, restless leg, and swelling were seen than in patients without pain. These findings may suggest that patients with $\mathrm{PD}$ who had a greater number of non-motor symptoms, such as weight loss, cognitive impairment, psychiatric problem, sleep disorder, and autonomic dysfunction, seemed to be at greater risk of pain occurrence. The neuronal degeneration in patients with pain may be more severe than patients without pain in basal ganglia as the disease progresses.

A logistic regression model comparing pain and multiple variables was established to help identify which variables may predict pain occurrence in patients with PD. HRSD and NMSQT scores were entered into the model. Dysregulation in dopamine signaling may directly and indirectly modulate the experience of pain by influencing affective and cognitive processes. Hypersensitivity to pain and high rates of comorbid chronic pain are common in disorders linked with deficits in the function of the dopamine system, including disorders of mood and affect, and PD [26]. Because there were no significant difference in the equivalent dosage of levodopa between patients with and without pain, these findings also suggest that common brain region such as the insular cortex, substantia nigra, amygdala, thalamus, prefrontal cortex, and anterior cingulated cortex, which affect cognitive function, emotional regulation, sleep, and autonomic functioning, may play a role in the occurrence of pain in patients with $\mathrm{PD}$ [31]. These non-motor symptoms became more prominent as the PD progressed. NMSQT score entered into the model revealed that the more non-motor symptoms, such as weight loss, memory disturbances, depression, insomnia, rapid eye movement disorder, and swelling, the patients had, the greater probability that pain would develop.

This study had several limitations. First, this was a cross-sectional study, which cannot determine the true risk of developing pain. Also, we cannot conclude whether the occurrence and degree of pain will change with disease progression. Second, although the daily equivalent levodopa dosage was calculated, different treatment groups were not established and the effect of different treatments and different drugs was not examined.

\section{Conclusion}

In summary, we found that pain in Chinese patients with PD is common. Non-motor symptoms were more prominent in patients with PD and pain, especially sensory, autonomic and neuropsychiatric symptoms, as well as sleep disorders, cognitive impairment, autonomic dysfunction, and weight loss. Patients with PD who had a greater number of non-motor symptoms, such as weight loss, cognitive impairment, psychiatric problems, sleep disorders, and autonomic dysfunction, seemed to be at higher risk of developing pain. Common brain region impairments affecting affective, cognitive, sleep and autonomic function may explain the common occurrence of pain in patients with PD.

\section{References}

1. Lee MA, Walker RW, Hildreth TJ, Prentice WM (2006) A survey of pain in idiopathic Parkinson's disease. J Pain Symptom Manage 32: 462-469.

2. Bair MJ, Robinson RL, Katon W, Kroenke K (2003) Depression and pain comorbidity: a literature review. Arch Intern Med 163: 2433-2445.

3. McWilliams LA, Cox BJ, Enns MW (2003) Mood and anxiety disorders associated with chronic pain: an examination in a nationally representative sample. Pain 106: 127-133.

4. Hanagasi HA, Akat S, Gurvit H, Yazici J, Emre M (2011) Pain is common in Parkinson's disease. Clin Neurol Neurosurg 113: 11-13.

5. Nieoullon A (2002) Dopamine and the regulation of cognition and attention. Prog Neurobiol 67: 53-83.

6. Schneier FR, Liebowitz MR, Abi-Dargham A, Zea-Ponce Y, Lin SH, et al. (2000) Low dopamine $\mathrm{D}(2)$ receptor binding potential in social phobia. Am J Psychiatry 157: 457-459.

7. Tiihonen J, Kuikka J, Bergström K, Lepola U, Koponen H, et al. (1997) Dopamine reuptake site densities in patients with social phobia. Am J Psychiatry 154: 239-242.

8. Dunlop BW, Nemeroff CB (2007) The role of dopamine in the pathophysiology of depression. Arch Gen Psychiatry 64: 327-337.

9. Roh JH, Kim BJ, Jang JH, Seo WK, Lee SH, et al. (2009) The relationship of pain and health-related quality of life in Korean patients with Parkinson's disease. Acta Neurol Scand 119: 397-403.

10. Hara T, Hirayama M, Mizutani Y, Hama T, Hori N, et al. (2013) Impaired pain processing in Parkinson's disease and its relative association with the sense of smell. Parkinsonism Relat Disord 19: 43-46.

11. Erro R, Santangelo G, Picillo M, Vitale C, Amboni M, et al. (2012) Link between non-motor symptoms and cognitive dysfunctions in de novo, drug-naive PD patients. J Neurol 259: 1808-1813.

12. Hughes AJ, Daniel SE, Kilford L, Lees AJ (1992) Accuracy of clinical diagnosis of idiopathic Parkinson's disease: a clinico-pathological study of 100 cases. J Neurol Neurosurg Psychiatry 55: 181-184. 
Citation: Mao CJ, Chen JP, Zhang XY, Li J, Xiong KP, Chen Y, Li SJ, Hu WD, et al., (2014) Prominent Non-Motor Symptoms in Patients with

13. Fahn S; Parkinson Study Group (2005) Does levodopa slow or hasten the rate of progression of Parkinson's disease? J Neurol 252 Suppl 4: IV37-37IV42.

14. Hoehn MM, Yahr MD (2001) Parkinsonism: onset, progression, and mortality. 1967. Neurology 57: S11-26.

15. Hamilton M (1960) A rating scale for depression. J Neurol Neurosurg Psychiatry 23: 56-62.

16. Nie K, Zhang Y, Wang L, Zhao J, Huang Z, et al. (2012) A pilot study of psychometric properties of the Beijing version of Montreal Cognitive Assessment in patients with idiopathic Parkinson's disease in China. J Clin Neurosci 19: 1497-1500.

17. Chaudhuri KR, Martinez-Martin P, Schapira AH, Stocchi F, Sethi K, et al. (2006) International multicenter pilot study of the first comprehensive self-completed nonmotor symptoms questionnaire for Parkinson's disease: the NMSQuest study. Mov Disord 21: 916-923.

18. Melzack R (1975) The McGill Pain Questionnaire: major properties and scoring methods. Pain 1: 277-299.

19. Cleeland CS, Ryan KM (1994) Pain assessment: global use of the Brief Pain Inventory. Ann Acad Med Singapore 23: 129-138.

20. Smeding HM, Esselink RA, Schmand B, Koning-Haanstra M, Nijhuis I, et al. (2005) Unilateral pallidotomy versus bilateral subthalamic nucleus stimulation in PD--a comparison of neuropsychological effects. J Neurol 252: 176-182.

21. Ford B (2010) Pain in Parkinson's disease. Mov Disord 25 Suppl 1: S98-103.

22. Airavaara M, Harvey BK, Voutilainen MH, Shen H, Chou J, et al. (2012) CDNF protects the nigrostriatal dopamine system and promotes recovery after MPTP treatment in mice. Cell Transplant 21: 1213-1223.
23. Ha AD, Jankovic J (2012) Pain in Parkinson's disease. Mov Disord 27: 485-491.

24. Santos-García D, Abella-Corral J, Aneiros-Díaz Á, Santos-Canelles H, Llaneza-González MA, et al. (2011) [Pain in Parkinson's disease: prevalence, characteristics, associated factors, and relation with other non motor symptoms, quality of life, autonomy, and caregiver burden]. Rev Neurol 52: 385-393.

25. Yong MH, Allen JC Jr, Prakash KM, Tan EK (2013) Differentiating nonmotor symptoms in Parkinson's disease from controls and hemifacial spasm. PLoS One 8: e49596.

26. Jarcho JM, Mayer EA, Jiang ZK, Feier NA, London ED (2012) Pain, affective symptoms, and cognitive deficits in patients with cerebral dopamine dysfunction. Pain 153: 744-754.

27. Mylius V, Brebbermann J, Dohmann H, Engau I, Oertel WH, et al. (2011) Pain sensitivity and clinical progression in Parkinson's disease. Mov Disord 26: 2220-2225.

28. Nutt JG (2007) Continuous dopaminergic stimulation: Is it the answer to the motor complications of Levodopa? Mov Disord 22: 1-9.

29. Lim SY, Farrell MJ, Gibson SJ, Helme RD, Lang AE, et al. (2008) Do dyskinesia and pain share common pathophysiological mechanisms in Parkinson's disease? Mov Disord 23: 1689-1695.

30. Romenets SR, Wolfson C, Galatas C, Pelletier A, Altman R, et al. (2012) Validation of the non-motor symptoms questionnaire (NMS-Quest). Parkinsonism Relat Disord 18: 54-58.

31. Raudino F (2001) Non motor off in Parkinson's disease. Acta Neurol Scand 104: 312-315. 